\title{
FUZZY BCI-SUBALGEBRAS WITH INTERVAL-VALUED MEMBERSHIP FUNCTIONS
}

\author{
SUNG MIN HONG, YOUNG BAE JUN, SEON JEONG KIM, and GWANG IL KIM
}

\author{
(Received 3 May 2000)
}

\begin{abstract}
The purpose of this paper is to define the notion of an interval-valued fuzzy BCI-subalgebra (briefly, an i-v fuzzy BCI-subalgebra) of a BCI-algebra. Necessary and sufficient conditions for an i-v fuzzy set to be an i-v fuzzy BCI-subalgebra are stated. A way to make a new i-v fuzzy BCI-subalgebra from old one is given. The images and inverse images of i-v fuzzy BCI-subalgebras are defined, and how the images or inverse images of i-v fuzzy BCI-subalgebras become i-v fuzzy BCI-subalgebras is studied.
\end{abstract}

2000 Mathematics Subject Classification. Primary 06F35, 03 B52.

1. Introduction. The notion of BCK-algebras was proposed by Iami and Iséki in 1966. In the same year, Iséki [2] introduced the notion of a BCI-algebra which is a generalization of a BCK-algebra. Since then numerous mathematical papers have been written investigating the algebraic properties of the BCK/BCI-algebras and their relationship with other universal structures including lattices and Boolean algebras. Fuzzy sets were initiated by Zadeh [3]. In [4], Zadeh made an extension of the concept of a fuzzy set by an interval-valued fuzzy set (i.e., a fuzzy set with an interval-valued membership function). This interval-valued fuzzy set is referred to as an i-v fuzzy set. In [4], Zadeh also constructed a method of approximate inference using his i-v fuzzy sets. In [1], Biswas defined interval-valued fuzzy subgroups (i.e., i-v fuzzy subgroups) of Rosenfeld's nature, and investigated some elementary properties. In this paper, using the notion of interval-valued fuzzy set by Zadeh, we introduce the concept of an interval-valued fuzzy BCI-subalgebra (briefly, i-v fuzzy BCI-subalgebra) of a BCI-algebra, and study some of their properties. Using an i-v level set of an i-v fuzzy set, we state a characterization of an i-v fuzzy BCI-subalgebra. We prove that every BCI-subalgebra of a BCI-algebra $X$ can be realized as an i-v level BCI-subalgebra of an i-v fuzzy BCI-subalgebra of $X$. In connection with the notion of homomorphism, we study how the images and inverse images of i-v fuzzy BCI-subalgebras become i-v fuzzy BCI-subalgebras.

2. Preliminaries. In this section, we include some elementary aspects that are necessary for this paper.

Recall that a $B C I$-algebra is an algebra $(X, *, 0)$ of type $(2,0)$ satisfying the following axioms:

(I) $((x * y) *(x * z)) *(z * y)=0$,

(II) $(x *(x * y)) * y=0$, 
(III) $x * x=0$, and

(IV) $x * y=0$ and $y * x=0$ imply $x=y$,

for every $x, y, z \in X$.

Note that the equality $0 *(x * y)=(0 * x) *(0 * y)$ holds in a BCI-algebra. A non-empty subset $S$ of a BCI-algebra $X$ is called a BCI-subalgebra of $X$ if $x * y \in S$ whenever $x, y \in S$. A mapping $f: X \rightarrow Y$ of BCI-algebras is called a homomorphism if $f(x * y)=f(x) * f(y)$ for all $x, y \in X$.

We now review some fuzzy logic concepts. Let $X$ be a set. A fuzzy set in $X$ is a function $\mu: X \rightarrow[0,1]$. Let $f$ be a mapping from a set $X$ into a set $Y$. Let $\nu$ be a fuzzy set in $Y$. Then the inverse image of $v$, denoted by $f^{-1}[v]$, is the fuzzy set in $X$ defined by $f^{-1}[\nu](x)=v(f(x))$ for all $x \in X$. Conversely, let $\mu$ be a fuzzy set in $X$. The image of $\mu$, written as $f[\mu]$, is a fuzzy set in $Y$ defined by

$$
f[\mu](y)= \begin{cases}\sup _{z \in f^{-1}(y)} \mu(z) & \text { if } f^{-1}(y) \neq \varnothing, \\ 0 & \text { otherwise }\end{cases}
$$

for all $y \in Y$, where $f^{-1}(y)=\{x \mid f(x)=y\}$.

An interval-valued fuzzy set (briefly, $i-v$ fuzzy set) $A$ defined on $X$ is given by

$$
\left.A=\left\{\left(x,\left[\mu_{A}^{L}(x), \mu_{A}^{U}(x)\right]\right)\right\}, \quad \forall x \in X \text { (briefly, denoted by } A=\left[\mu_{A}^{L}, \mu_{A}^{U}\right]\right),
$$

where $\mu_{A}^{L}$ and $\mu_{A}^{U}$ are two fuzzy sets in $X$ such that $\mu_{A}^{L}(x) \leq \mu_{A}^{U}(x)$ for all $x \in X$.

Let $\bar{\mu}_{A}(x)=\left[\mu_{A}^{L}(x), \mu_{A}^{U}(x)\right], \forall x \in X$ and let $D[0,1]$ denotes the family of all closed subintervals of $[0,1]$. If $\mu_{A}^{L}(x)=\mu_{A}^{U}(x)=c$, say, where $0 \leq c \leq 1$, then we have $\bar{\mu}_{A}(x)=$ $[c, c]$ which we also assume, for the sake of convenience, to belong to $D[0,1]$. Thus $\bar{\mu}_{A}(x) \in D[0,1], \forall x \in X$, and therefore the i-v fuzzy set $A$ is given by

$$
A=\left\{\left(x, \bar{\mu}_{A}(x)\right)\right\}, \quad \forall x \in X, \text { where } \bar{\mu}_{A}: X \rightarrow D[0,1] .
$$

Now let us define what is known as refined minimum (briefly, rmin) of two elements in $D[0,1]$. We also define the symbols " $\geq$ ", " $\leq$ ", and "=" in case of two elements in $D[0,1]$. Consider two elements $D_{1}:=\left[a_{1}, b_{1}\right]$ and $D_{2}:=\left[a_{2}, b_{2}\right] \in D[0,1]$. Then

$$
\begin{aligned}
& \operatorname{rmin}\left(D_{1}, D_{2}\right)=\left[\min \left\{a_{1}, a_{2}\right\}, \min \left\{b_{1}, b_{2}\right\}\right] \\
& D_{1} \geq D_{2} \text { if and only if } a_{1} \geq a_{2}, b_{1} \geq b_{2}
\end{aligned}
$$

and similarly we may have $D_{1} \leq D_{2}$ and $D_{1}=D_{2}$.

DEFINITION 2.1. A fuzzy set $\mu$ in a BCI-algebra $X$ is called a fuzzy BCI-subalgebra of $X$ if $\mu(x * y) \geq \min \{\mu(x), \mu(y)\}$ for all $x, y \in X$.

3. Interval-valued fuzzy BCI-subalgebras. In what follows, let $X$ denote a BCIalgebra unless otherwise specified. We begin with the following two propositions. 
Proposition 3.1. Let $f$ be a homomorphism from a BCI-algebra $X$ into a BCIalgebra $Y$. If $v$ is a fuzzy BCI-subalgebra of $Y$, then the inverse image $f^{-1}[v]$ of $v$ is a fuzzy BCI-subalgebra of $X$.

Proof. For any $x, y \in X$, we have

$$
\begin{aligned}
f^{-1}[v](x * y) & =v(f(x * y))=v(f(x) * f(y)) \\
& \geq \min \{v(f(x)), v(f(y))\} \\
& =\min \left\{f^{-1}[v](x), f^{-1}[v](y)\right\} .
\end{aligned}
$$

Hence $f^{-1}[v]$ is a fuzzy BCI-subalgebra of $X$.

Proposition 3.2. Let $f: X \rightarrow Y$ be a homomorphism between BCI-algebras $X$ and $Y$. For every fuzzy BCI-subalgebra $\mu$ of $X$, the image $f[\mu]$ of $\mu$ is a fuzzy $B C I$-subalgebra of $Y$.

Proof. We first prove that

$$
f^{-1}\left(y_{1}\right) * f^{-1}\left(y_{2}\right) \subseteq f^{-1}\left(y_{1} * y_{2}\right)
$$

for all $y_{1}, y_{2} \in Y$. For, if $x \in f^{-1}\left(y_{1}\right) * f^{-1}\left(y_{2}\right)$, then $x=x_{1} * x_{2}$ for some $x_{1} \in$ $f^{-1}\left(y_{1}\right)$ and $x_{2} \in f^{-1}\left(y_{2}\right)$. Since $f$ is a homomorphism, it follows that $f(x)=$ $f\left(x_{1} * x_{2}\right)=f\left(x_{1}\right) * f\left(x_{2}\right)=y_{1} * y_{2}$ so that $x \in f^{-1}\left(y_{1} * y_{2}\right)$. Hence (3.2) holds. Now let $y_{1}, y_{2} \in Y$ be arbitrarily given. Assume that $y_{1} * y_{2} \notin \operatorname{Im}(f)$. Then $f[\mu]\left(y_{1} * y_{2}\right)=$ 0 . But if $y_{1} * y_{2} \notin \operatorname{Im}(f)$, that is, $f^{-1}\left(y_{1} * y_{2}\right)=\varnothing$, then $f^{-1}\left(y_{1}\right)=\varnothing$ or $f^{-1}\left(y_{2}\right)=\varnothing$ by (3.2). Thus $f[\mu]\left(y_{1}\right)=0$ or $f[\mu]\left(y_{2}\right)=0$, and so

$$
f[\mu]\left(y_{1} * y_{2}\right)=0=\min \left\{f[\mu]\left(y_{1}\right), f[\mu]\left(y_{2}\right)\right\} .
$$

Suppose that $f^{-1}\left(y_{1} * y_{2}\right) \neq \varnothing$. Then we should consider the two cases:

$$
\begin{aligned}
& f^{-1}\left(y_{1}\right)=\varnothing \quad \text { or } \quad f^{-1}\left(y_{2}\right)=\varnothing \text {, } \\
& f^{-1}\left(y_{1}\right) \neq \varnothing \quad \text { and } \quad f^{-1}\left(y_{2}\right) \neq \varnothing \text {. }
\end{aligned}
$$

For the case (3.4), we have $f[\mu]\left(y_{1}\right)=0$ or $f[\mu]\left(y_{2}\right)=0$, and so

$$
f[\mu]\left(y_{1} * y_{2}\right) \geq 0=\min \left\{f[\mu]\left(y_{1}\right), f[\mu]\left(y_{2}\right)\right\} .
$$

Case (3.5) implies, from (3.2), that

$$
\begin{aligned}
f[\mu]\left(y_{1} * y_{2}\right) & =\sup _{z \in f^{-1}\left(y_{1} * y_{2}\right)} \mu(z) \geq \sup _{z \in f^{-1}\left(y_{1}\right) * f^{-1}\left(y_{2}\right)} \mu(z) \\
& =\sup _{x_{1} \in f^{-1}\left(y_{1}\right), x_{2} \in f^{-1}\left(y_{2}\right)} \mu\left(x_{1} * x_{2}\right) .
\end{aligned}
$$


Since $\mu$ is a fuzzy BCI-subalgebra of $X$, it follows from the definition of a fuzzy BCIsubalgebra that

$$
\begin{aligned}
f[\mu]\left(y_{1} * y_{2}\right) \geq & \sup _{x_{1} \in f^{-1}\left(y_{1}\right), x_{2} \in f^{-1}\left(y_{2}\right)} \min \left\{\mu\left(x_{1}\right), \mu\left(x_{2}\right)\right\} \\
& =\sup _{x_{1} \in f^{-1}\left(y_{1}\right)}\left(\min \left\{\sup _{x_{2} \in f^{-1}\left(y_{2}\right)} \mu\left(x_{1}\right), \mu\left(x_{2}\right)\right\}\right) \\
& =\sup _{x_{1} \in f^{-1}\left(y_{1}\right)}\left(\min \left\{\mu\left(x_{1}\right), \sup _{x_{2} \in f^{-1}\left(y_{2}\right)} \mu\left(x_{2}\right)\right\}\right) \\
& =\sup _{x_{1} \in f^{-1}\left(y_{1}\right)}\left(\min \left\{\mu\left(x_{1}\right), f[\mu]\left(y_{2}\right)\right\}\right) \\
& =\min \left\{\sup _{x_{1} \in f^{-1}\left(y_{1}\right)} \mu\left(x_{1}\right), f[\mu]\left(y_{2}\right)\right\} \\
& =\min \left\{f[\mu]\left(y_{1}\right), f[\mu]\left(y_{2}\right)\right\} .
\end{aligned}
$$

Hence $f[\mu]\left(y_{1} * y_{2}\right) \geq \min \left\{f[\mu]\left(y_{1}\right), f[\mu]\left(y_{2}\right)\right\}$ for all $y_{1}, y_{2} \in Y$. This completes the proof.

DefinITION 3.3. An i-v fuzzy set $A$ in $X$ is called an interval-valued fuzzy BCIsubalgebra (briefly, $i$-v fuzzy BCI-subalgebra) of $X$ if

$$
\bar{\mu}_{A}(x * y) \geq \operatorname{rmin}\left\{\bar{\mu}_{A}(x), \bar{\mu}_{A}(y)\right\} \quad \forall x, y \in X .
$$

EXAMPLE 3.4. Let $X=\{0, a, b, c\}$ be a BCI-algebra with the following Cayley table:

TABLE 3.1.

\begin{tabular}{l|l|l|l|l}
\hline$*$ & 0 & $a$ & $b$ & $c$ \\
\hline 0 & 0 & $c$ & 0 & $a$ \\
$a$ & $a$ & 0 & $a$ & $c$ \\
$b$ & $b$ & $c$ & 0 & $a$ \\
$c$ & $c$ & $a$ & $c$ & 0 \\
\hline
\end{tabular}

let an i-v fuzzy set $A$ defined on $X$ be given by

$$
\bar{\mu}_{A}(x)= \begin{cases}{[0.2,0.8]} & \text { if } x \in\{0, b\}, \\ {[0.1,0.7]} & \text { otherwise. }\end{cases}
$$

It is easy to check that $A$ is an i-v fuzzy BCI-subalgebra of $X$.

LEMMA 3.5. If $A$ is an $i$-v fuzzy BCI-subalgebra of $X$, then $\bar{\mu}_{A}(0) \geq \bar{\mu}_{A}(x)$ for all $x \in X$. 
Proof. For every $x \in X$, we have

$$
\begin{aligned}
\bar{\mu}_{A}(0) & =\bar{\mu}_{A}(x * x) \geq \operatorname{rmin}\left\{\bar{\mu}_{A}(x), \bar{\mu}_{A}(x)\right\} \\
& =\operatorname{rmin}\left\{\left[\mu_{A}^{L}(x), \mu_{A}^{U}(x)\right],\left[\mu_{A}^{L}(x), \mu_{A}^{U}(x)\right]\right\} \\
& =\left[\mu_{A}^{L}(x), \mu_{A}^{U}(x)\right]=\bar{\mu}_{A}(x),
\end{aligned}
$$

this completes the proof.

THEOREM 3.6. Let $A$ be an $i$-v fuzzy BCI-subalgebra of $X$. If there is a sequence $\left\{x_{n}\right\}$ in $X$ such that

$$
\lim _{n \rightarrow \infty} \bar{\mu}_{A}\left(x_{n}\right)=[1,1]
$$

then $\bar{\mu}_{A}(0)=[1,1]$.

Proof. Since $\bar{\mu}_{A}(0) \geq \bar{\mu}_{A}(x)$ for all $x \in X$, we have $\bar{\mu}_{A}(0) \geq \bar{\mu}_{A}\left(x_{n}\right)$ for every positive integer $n$. Note that

$$
[1,1] \geq \bar{\mu}_{A}(0) \geq \lim _{n \rightarrow \infty} \bar{\mu}_{A}\left(x_{n}\right)=[1,1]
$$

Hence $\bar{\mu}_{A}(0)=[1,1]$.

THEOREM 3.7. An i-v fuzzy set $A=\left[\mu_{A}^{L}, \mu_{A}^{U}\right]$ in $X$ is an i-v fuzzy BCI-subalgebra of $X$ if and only if $\mu_{A}^{L}$ and $\mu_{A}^{U}$ are fuzzy BCI-subalgebras of $X$.

Proof. Suppose that $\mu_{A}^{L}$ and $\mu_{A}^{U}$ are fuzzy BCI-subalgebras of $X$. Let $x, y \in X$. Then

$$
\begin{aligned}
\bar{\mu}_{A}(x * y) & =\left[\mu_{A}^{L}(x * y), \mu_{A}^{U}(x * y)\right] \\
& \geq\left[\min \left\{\mu_{A}^{L}(x), \mu_{A}^{L}(y)\right\}, \min \left\{\mu_{A}^{U}(x), \mu_{A}^{U}(y)\right\}\right] \\
& =\operatorname{rmin}\left\{\left[\mu_{A}^{L}(x), \mu_{A}^{U}(x)\right],\left[\mu_{A}^{L}(y), \mu_{A}^{U}(y)\right]\right\} \\
& =\operatorname{rmin}\left\{\bar{\mu}_{A}(x), \bar{\mu}_{A}(y)\right\} .
\end{aligned}
$$

Hence $A$ is an i-v fuzzy BCI-subalgebra of $X$.

Conversely, assume that $A$ is an i-v fuzzy BCI-subalgebra of $X$. For any $x, y \in X$, we have

$$
\begin{aligned}
{\left[\mu_{A}^{L}(x * y), \mu_{A}^{U}(x * y)\right] } & =\bar{\mu}_{A}(x * y) \geq \operatorname{rmin}\left\{\bar{\mu}_{A}(x), \bar{\mu}_{A}(y)\right\} \\
& =\operatorname{rmin}\left\{\left[\mu_{A}^{L}(x), \mu_{A}^{U}(x)\right],\left[\mu_{A}^{L}(y), \mu_{A}^{U}(y)\right]\right\} \\
& =\left[\min \left\{\mu_{A}^{L}(x), \mu_{A}^{L}(y)\right\}, \min \left\{\mu_{A}^{U}(x), \mu_{A}^{U}(y)\right\}\right] .
\end{aligned}
$$

It follows that $\mu_{A}^{L}(x * y) \geq \min \left\{\mu_{A}^{L}(x), \mu_{A}^{L}(y)\right\}$ and $\mu_{A}^{U}(x * y) \geq \min \left\{\mu_{A}^{U}(x), \mu_{A}^{U}(y)\right\}$. Hence $\mu_{A}^{L}$ and $\mu_{A}^{U}$ are fuzzy BCI-subalgebras of $X$.

THEOREM 3.8. Let $A$ be an i-v fuzzy set in $X$. Then $A$ is an i-v fuzzy BCI-subalgebra of $X$ if and only if the nonempty set

$$
U\left(A ;\left[\delta_{1}, \delta_{2}\right]\right):=\left\{x \in X \mid \bar{\mu}_{A}(x) \geq\left[\delta_{1}, \delta_{2}\right]\right\}
$$

is a BCI-subalgebra of $X$ for every $\left[\delta_{1}, \delta_{2}\right] \in D[0,1]$. 
We then call $\bar{U}\left(A ;\left[\delta_{1}, \delta_{2}\right]\right)$ the $i$-v level BCI-subalgebra of $A$.

Proof. Assume that $A$ is an i-v fuzzy BCI-subalgebra of $X$ and let $\left[\delta_{1}, \delta_{2}\right] \in D[0,1]$ be such that $x, y \in \bar{U}\left(A ;\left[\delta_{1}, \delta_{2}\right]\right)$. Then

$$
\bar{\mu}_{A}(x * y) \geq \operatorname{rmin}\left\{\bar{\mu}_{A}(x), \bar{\mu}_{A}(y)\right\} \geq \operatorname{rmin}\left\{\left[\delta_{1}, \delta_{2}\right],\left[\delta_{1}, \delta_{2}\right]\right\}=\left[\delta_{1}, \delta_{2}\right],
$$

and so $x * y \in \bar{U}\left(A ;\left[\delta_{1}, \delta_{2}\right]\right)$. Thus $\bar{U}\left(A ;\left[\delta_{1}, \delta_{2}\right]\right)$ is a BCI-subalgebra of $X$.

Conversely, assume that $\bar{U}\left(A ;\left[\delta_{1}, \delta_{2}\right]\right)(\neq \varnothing)$ is a BCI-subalgebra of $X$ for every $\left[\delta_{1}, \delta_{2}\right] \in D[0,1]$. Suppose there exist $x_{0}, y_{0} \in X$ such that

$$
\bar{\mu}_{A}\left(x_{0} * y_{0}\right)<\operatorname{rmin}\left\{\bar{\mu}_{A}\left(x_{0}\right), \bar{\mu}_{A}\left(y_{0}\right)\right\} .
$$

Let $\bar{\mu}_{A}\left(x_{0}\right)=\left[\gamma_{1}, \gamma_{2}\right], \bar{\mu}_{A}\left(y_{0}\right)=\left[\gamma_{3}, \gamma_{4}\right]$, and $\bar{\mu}_{A}\left(x_{0} * y_{0}\right)=\left[\delta_{1}, \delta_{2}\right]$. Then

$$
\left[\delta_{1}, \delta_{2}\right]<\operatorname{rmin}\left\{\left[\gamma_{1}, \gamma_{2}\right],\left[\gamma_{3}, \gamma_{4}\right]\right\}=\left[\min \left\{\gamma_{1}, \gamma_{3}\right\}, \min \left\{\gamma_{2}, \gamma_{4}\right\}\right] .
$$

Hence $\delta_{1}<\min \left\{\gamma_{1}, \gamma_{3}\right\}$ and $\delta_{2}<\min \left\{\gamma_{2}, \gamma_{4}\right\}$. Taking

$$
\left[\lambda_{1}, \lambda_{2}\right]=\frac{1}{2}\left(\bar{\mu}_{A}\left(x_{0} * y_{0}\right)+\operatorname{rmin}\left\{\bar{\mu}_{A}\left(x_{0}\right), \bar{\mu}_{A}\left(y_{0}\right)\right\}\right),
$$

we obtain

$$
\begin{aligned}
{\left[\lambda_{1}, \lambda_{2}\right] } & =\frac{1}{2}\left(\left[\delta_{1}, \delta_{2}\right]+\left[\min \left\{\gamma_{1}, \gamma_{3}\right\}, \min \left\{\gamma_{2}, \gamma_{4}\right\}\right]\right) \\
& =\left[\frac{1}{2}\left(\delta_{1}+\min \left\{\gamma_{1}, \gamma_{3}\right\}\right), \frac{1}{2}\left(\delta_{2}+\min \left\{\gamma_{2}, \gamma_{4}\right\}\right)\right]
\end{aligned}
$$

It follows that

$$
\begin{aligned}
& \min \left\{\gamma_{1}, \gamma_{3}\right\}>\lambda_{1}=\frac{1}{2}\left(\delta_{1}+\min \left\{\gamma_{1}, \gamma_{3}\right\}\right)>\delta_{1}, \\
& \min \left\{\gamma_{2}, \gamma_{4}\right\}>\lambda_{2}=\frac{1}{2}\left(\delta_{2}+\min \left\{\gamma_{2}, \gamma_{4}\right\}\right)>\delta_{2}
\end{aligned}
$$

so that $\left[\min \left\{\gamma_{1}, \gamma_{3}\right\}, \min \left\{\gamma_{2}, \gamma_{4}\right\}\right]>\left[\lambda_{1}, \lambda_{2}\right]>\left[\delta_{1}, \delta_{2}\right]=\bar{\mu}_{A}\left(x_{0} * y_{0}\right)$. Therefore, $x_{0} * y_{0} \notin \bar{U}\left(A ;\left[\lambda_{1}, \lambda_{2}\right]\right)$. On the other hand,

$$
\begin{aligned}
& \bar{\mu}_{A}\left(x_{0}\right)=\left[\gamma_{1}, \gamma_{2}\right] \geq\left[\min \left\{\gamma_{1}, \gamma_{3}\right\}, \min \left\{\gamma_{2}, \gamma_{4}\right\}\right]>\left[\lambda_{1}, \lambda_{2}\right], \\
& \bar{\mu}_{A}\left(y_{0}\right)=\left[\gamma_{3}, \gamma_{4}\right] \geq\left[\min \left\{\gamma_{1}, \gamma_{3}\right\}, \min \left\{\gamma_{2}, \gamma_{4}\right\}\right]>\left[\lambda_{1}, \lambda_{2}\right],
\end{aligned}
$$

and so $x_{0}, y_{0} \in \bar{U}\left(A ;\left[\lambda_{1}, \lambda_{2}\right]\right)$. It contradicts that $\bar{U}\left(A ;\left[\lambda_{1}, \lambda_{2}\right]\right)$ is a BCI-subalgebra of $X$. Hence $\bar{\mu}_{A}(x * y) \geq \operatorname{rmin}\left\{\bar{\mu}_{A}(x), \bar{\mu}_{A}(y)\right\}$ for all $x, y \in X$. This completes the proof.

THEOREM 3.9. Every BCI-subalgebra of $X$ can be realized as an i-v level BCI-subalgebra of an i-v fuzzy BCI-subalgebra of $X$.

Proof. Let $Y$ be a BCI-subalgebra of $X$ and let $A$ be an i-v fuzzy set on $X$ defined by

$$
\bar{\mu}_{A}(x)= \begin{cases}{\left[\alpha_{1}, \alpha_{2}\right]} & \text { if } x \in Y, \\ {[0,0]} & \text { otherwise }\end{cases}
$$

where $\alpha_{1}, \alpha_{2} \in(0,1]$ with $\alpha_{1}<\alpha_{2}$. It is clear that $\bar{U}\left(A ;\left[\alpha_{1}, \alpha_{2}\right]\right)=Y$. We show that $A$ 
is an i-v fuzzy BCI-subalgebra of $X$. Let $x, y \in X$. If $x, y \in Y$, then $x * y \in Y$ and so

$$
\bar{\mu}_{A}(x * y)=\left[\alpha_{1}, \alpha_{2}\right]=\operatorname{rmin}\left\{\left[\alpha_{1}, \alpha_{2}\right],\left[\alpha_{1}, \alpha_{2}\right]\right\}=\operatorname{rmin}\left\{\bar{\mu}_{A}(x), \bar{\mu}_{A}(y)\right\} .
$$

If $x, y \notin Y$, then $\bar{\mu}_{A}(x)=[0,0]=\bar{\mu}_{A}(y)$ and thus

$$
\bar{\mu}_{A}(x * y) \geq[0,0]=\operatorname{rmin}\{[0,0],[0,0]\}=\operatorname{rmin}\left\{\bar{\mu}_{A}(x), \bar{\mu}_{A}(y)\right\} .
$$

If $x \in Y$ and $y \notin Y$, then $\bar{\mu}_{A}(x)=\left[\alpha_{1}, \alpha_{2}\right]$ and $\bar{\mu}_{A}(y)=[0,0]$. It follows that

$$
\bar{\mu}_{A}(x * y) \geq[0,0]=\operatorname{rmin}\left\{\left[\alpha_{1}, \alpha_{2}\right],[0,0]\right\}=\operatorname{rmin}\left\{\bar{\mu}_{A}(x), \bar{\mu}_{A}(y)\right\} .
$$

Similarly for the case $x \notin Y$ and $y \in Y$, we get $\bar{\mu}_{A}(x * y) \geq \operatorname{rmin}\left\{\bar{\mu}_{A}(x), \bar{\mu}_{A}(y)\right\}$. Therefore $A$ is an i-v fuzzy BCI-subalgebra of $X$, and the proof is complete.

THEOREM 3.10. Let $Y$ be a subset of $X$ and let $A$ be an $i-v$ fuzzy set on $X$ which is given in the proof of Theorem 3.9. If $A$ is an $i-v$ fuzzy BCI-subalgebra of $X$, then $Y$ is a BCI-subalgebra of $X$.

Proof. Assume that $A$ is an i-v fuzzy BCI-subalgebra of $X$. Let $x, y \in Y$. Then $\bar{\mu}_{A}(x)=\left[\alpha_{1}, \alpha_{2}\right]=\bar{\mu}_{A}(y)$, and so

$$
\bar{\mu}_{A}(x * y) \geq \operatorname{rmin}\left\{\bar{\mu}_{A}(x), \bar{\mu}_{A}(y)\right\}=\operatorname{rmin}\left\{\left[\alpha_{1}, \alpha_{2}\right],\left[\alpha_{1}, \alpha_{2}\right]\right\}=\left[\alpha_{1}, \alpha_{2}\right] .
$$

This implies that $x * y \in Y$. Hence $Y$ is a BCI-subalgebra of $X$.

THEOREM 3.11. If $A$ is an $i-v$ fuzzy BCI-subalgebra of $X$, then the set

$$
X_{\bar{\mu}_{A}}:=\left\{x \in X \mid \bar{\mu}_{A}(x)=\bar{\mu}_{A}(0)\right\}
$$

is a BCI-subalgebra of $X$.

Proof. Let $x, y \in X_{\bar{\mu}_{A}}$. Then $\bar{\mu}_{A}(x)=\bar{\mu}_{A}(0)=\bar{\mu}_{A}(y)$, and so

$$
\bar{\mu}_{A}(x * y) \geq \operatorname{rmin}\left\{\bar{\mu}_{A}(x), \bar{\mu}_{A}(y)\right\}=\operatorname{rmin}\left\{\bar{\mu}_{A}(0), \bar{\mu}_{A}(0)\right\}=\bar{\mu}_{A}(0) .
$$

Combining this and Lemma 3.5, we get $\bar{\mu}_{A}(x * y)=\bar{\mu}_{A}(0)$, that is, $x * y \in X_{\bar{\mu}_{A}}$. Hence $X_{\bar{\mu}_{A}}$ is a BCI-subalgebra of $X$.

The following is a way to make a new i-v fuzzy BCI-subalgebra from old one.

THEOREM 3.12. For an $i$-v fuzzy BCI-subalgebra $A$ of $X$, the $i$-v fuzzy set $A^{*}$ in $X$ defined by $\bar{\mu}_{A^{*}}(x)=\bar{\mu}_{A}(0 * x)$ for all $x \in X$ is an i-v fuzzy BCI-subalgebra of $X$.

Proof. Since the equality $0 *(x * y)=(0 * x) *(0 * y)$ holds for all $x, y \in X$, we have

$$
\begin{aligned}
\bar{\mu}_{A^{*}}(x * y) & =\bar{\mu}_{A}(0 *(x * y))=\bar{\mu}_{A}((0 * x) *(0 * y)) \\
& \geq \operatorname{rmin}\left\{\bar{\mu}_{A}(0 * x), \bar{\mu}_{A}(0 * y)\right\} \\
& =\operatorname{rmin}\left\{\bar{\mu}_{A^{*}}(x), \bar{\mu}_{A^{*}}(y)\right\}
\end{aligned}
$$

for all $x, y \in X$. Therefore $A^{*}$ is an i-v fuzzy BCI-subalgebra of $X$. 
Definition 3.13 (Biswas [1]). Let $f$ be a mapping from a set $X$ into a set $Y$. Let $B$ be an i-v fuzzy set in $Y$. Then the inverse image of $B$, denoted by $f^{-1}[B]$, is the i-v fuzzy set in $X$ with the membership function given by $\bar{\mu}_{f^{-1}[B]}(x)=\bar{\mu}_{B}(f(x))$ for all $x \in X$.

LEMMA 3.14 (Biswas [1]). Let $f$ be a mapping from a set $X$ into a set $Y$. Let $m=$ $\left[m^{L}, m^{U}\right]$ and $n=\left[n^{L}, n^{U}\right]$ be i-v fuzzy sets in $X$ and $Y$, respectively. Then

(i) $f^{-1}(n)=\left[f^{-1}\left(n^{L}\right), f^{-1}\left(n^{U}\right)\right]$,

(ii) $f(m)=\left[f\left(m^{L}\right), f\left(m^{U}\right)\right]$.

THEOREM 3.15. Let $f$ be a homomorphism from a BCI-algebra $X$ into a BCI-algebra $Y$. If $B$ is an $i-v$ fuzzy BCI-subalgebra of $Y$, then the inverse image $f^{-1}[B]$ of $B$ is an $i-v$ fuzzy BCI-subalgebra of $X$.

Proof. Since $B=\left[\mu_{B}^{L}, \mu_{B}^{U}\right]$ is an i-v fuzzy BCI-subalgebra of $Y$, it follows from Theorem 3.7 that $\mu_{B}^{L}$ and $\mu_{B}^{U}$ are fuzzy BCI-subalgebras of $Y$. Using Proposition 3.1, we know that $f^{-1}\left[\mu_{B}^{L}\right]$ and $f^{-1}\left[\mu_{B}^{U}\right]$ are fuzzy BCI-subalgebras of $X$. Hence, by Lemma 3.14 and Theorem 3.7, we conclude that $f^{-1}[B]=\left[f^{-1}\left[\mu_{B}^{L}\right], f^{-1}\left[\mu_{B}^{U}\right]\right]$ is an i-v fuzzy BCIsubalgebra of $X$.

Definition 3.16 (Biswas [1]). Let $f$ be a mapping from a set $X$ into a set $Y$. Let $A$ be an i-v fuzzy set in $X$. Then the image of $A$, denoted by $f[A]$, is the i-v fuzzy set in $Y$ with the membership function defined by

$$
\bar{\mu}_{f[A]}(y)= \begin{cases}\operatorname{rsup}_{z \in f^{-1}(y)} \bar{\mu}_{A}(z) & \text { if } f^{-1}(y) \neq \varnothing, \forall y \in Y, \\ {[0,0]} & \text { otherwise, }\end{cases}
$$

where $f^{-1}(y)=\{x \mid f(x)=y\}$.

THEOREM 3.17. Let $f$ be a homomorphism from a BCI-algebra $X$ into a BCI-algebra $Y$. If $A$ is an $i-v$ fuzzy BCI-subalgebra of $X$, then the image $f[A]$ of $A$ is an $i-v$ fuzzy BCI-subalgebra of $Y$.

Proof. Assume that $A$ is an i-v fuzzy BCI-subalgebra of $X$. Note that $A=\left[\mu_{A}^{L}, \mu_{A}^{U}\right]$ is an i-v fuzzy BCI-subalgebra of $X$ if and only if $\mu_{A}^{L}$ and $\mu_{A}^{U}$ are fuzzy BCI-subalgebras of $X$. It follows from Proposition 3.2 that the images $f\left[\mu_{A}^{L}\right]$ and $f\left[\mu_{A}^{U}\right]$ are fuzzy BCIsubalgebras of $Y$. Combining Theorem 3.7 and Lemma 3.14, we conclude that $f[A]=$ $\left[f\left[\mu_{A}^{L}\right], f\left[\mu_{A}^{U}\right]\right]$ is an i-v fuzzy BCI-subalgebra of $Y$.

ACKNOWLEDGEMENT. This work was supported by Korea Research Foundation Grant KRF-99-005-D00003.

\section{REFERENCES}

[1] R. Biswas, Rosenfeld's fuzzy subgroups with interval-valued membership functions, Fuzzy Sets and Systems 63 (1994), no. 1, 87-90. CMP 1273 001. Zbl 844.20060.

[2] K. Iséki, An algebra related with a propositional calculus, Proc. Japan Acad. 42 (1966), 26-29. MR 34\#2433. Zbl 207.29304.

[3] L. A. Zadeh, Fuzzy sets, Information and Control 8 (1965), 338-353. MR 36\#2509. Zbl 139.24606. 
[4] , The concept of a linguistic variable and its application to approximate reasoning. I, Information Sci. 8 (1975), 199-249. MR 52\#7225a. Zbl 397.68071.

Sung Min Hong: Department of Mathematics, Gyeongsang National University, CHINJU 660-701, KOREA

E-mail address: smhong@nongae.gsnu.ac.kr

Young BAe Jun: Department of MATHematics Education, GyeONGSANG NATional UniVERSITY, CHINJU 660-701, KOREA

E-mail address: ybjun@nongae.gsnu.ac. $\mathrm{kr}$

Seon Jeong Kim: Department of Mathematics, Gyeongsang National University, CHINJu 660-701, KoreA

E-mail address: skim@nongae.gsnu.ac.kr

GWANG Il Kim: DePARTMENT OF MATHEMATICS, GyeONGSANG NATIONAL UNIVERSiTy, ChinJU 660-701, KOREA

E-mail address: gikim@nongae.gsnu.ac.kr 


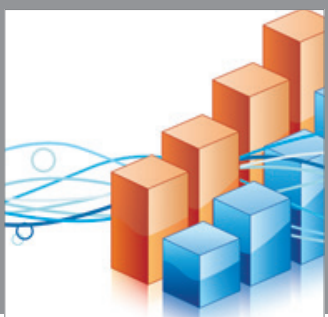

Advances in

Operations Research

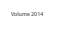

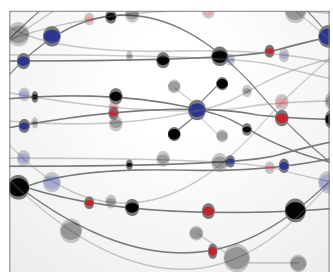

\section{The Scientific} World Journal
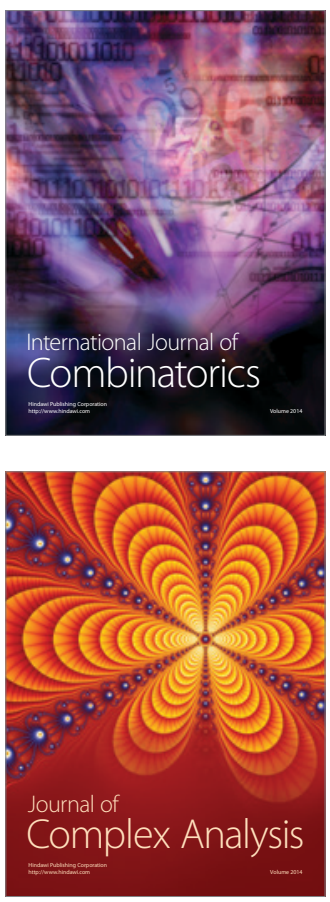

International Journal of

Mathematics and

Mathematical

Sciences
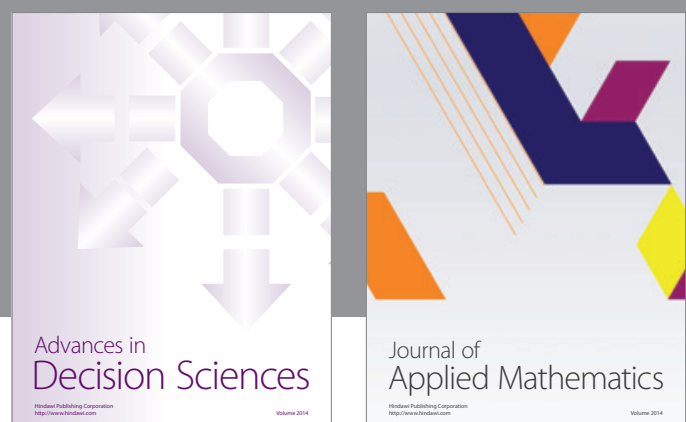

Journal of

Applied Mathematics
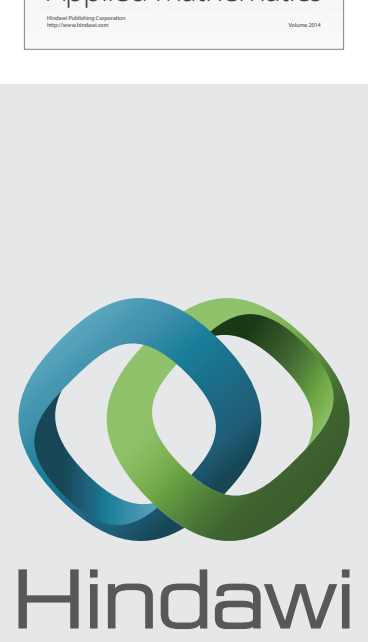

Submit your manuscripts at http://www.hindawi.com
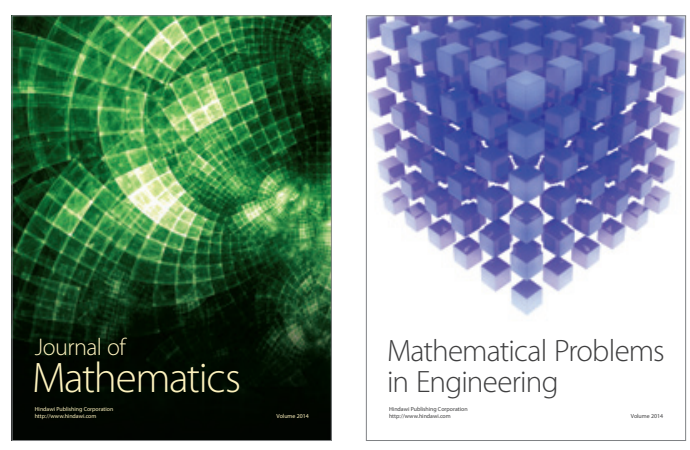

Mathematical Problems in Engineering
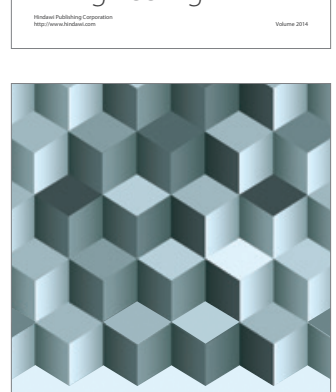

Journal of

Function Spaces
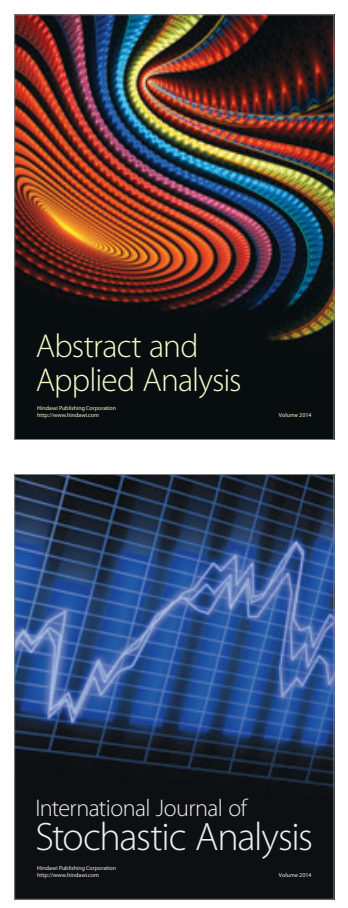

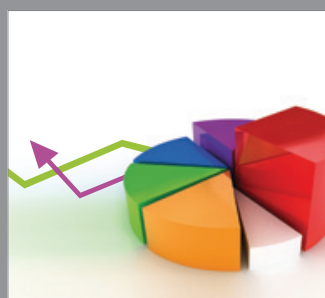

ournal of

Probability and Statistics

Promensencen
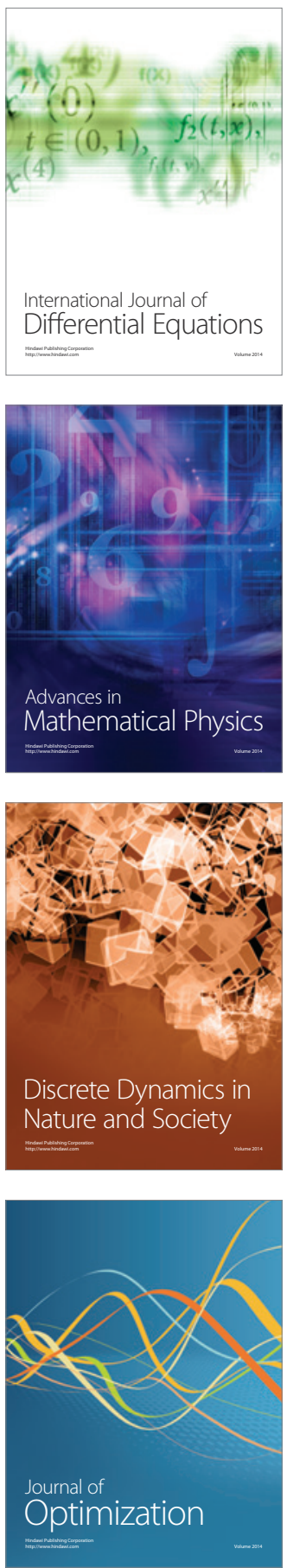